Bull. Korean Math. Soc. 52 (2015), No. 3, pp. 735-740

http://dx.doi.org/10.4134/BKMS.2015.52.3.735

\title{
AN ESTIMATE OF HEMPEL DISTANCE FOR BRIDGE SPHERES
}

\author{
AyAKo IDO
}

\begin{abstract}
Tomova [8] gave an upper bound for the distance of a bridge surface for a knot with two different bridge positions in a 3-manifold. In this paper, we show that the result of Tomova [8, Theorem 10.3] can be improved in the case when there are two different bridge spheres for a link in $S^{3}$.
\end{abstract}

\section{Introduction}

Hempel [4] introduced the concept of distance of a Heegaard surface, and it is shown by many authors that it well represents various complexities of 3manifolds. For example, Hartshorn [2] showed that the Euler characteristic of an incompressible surface in a 3-manifold bounds the distance of its Heegaard splittings, and Scharlemann and Tomova [7] showed that the Euler characteristic of any Heegaard splitting of a 3-manifold similarly bounds the distance of any non-isotopic Heegaard splitting.

The above concept and results have been extended to bridge surfaces for knots and links in closed 3-manifolds, and have been studied by several authors. For example, Bachman and Schleimer [1] proved that Hartshorn's results can be extended to the distance of a bridge surface for a knot in a closed orientable 3-manifold, and also Tomova [8] proved that Scharlemann and Tomova's results can be extended to the distance of a bridge surface for a knot in a closed orientable 3-manifold. Moreover, Johnson and Tomova [6] proved that Tomova's result can be extended to a bridge surface for a tangle in a compact 3-manifold. Recently Jang [5] showed that for a link in a closed orientable 3-manifold, the result of Bachman and Schleimer [1] can be improved in the case when there exist essential meridional spheres.

In this paper, we find a property of essential simple closed curves disjoint from the disk complex of a 3-ball containing trivial arcs (for detail, see Lemma 2.1). This allows us to improve the result of Tomova [8, Theorem 10.3] in the case when there are two different bridge spheres for a link in $S^{3}$.

Received March 6, 2013; Revised August 10, 2013.

2010 Mathematics Subject Classification. 57M27.

Key words and phrases. Heegaard splitting, bridge decomposition, distance. 
Theorem 1.1. Suppose $L$ is a link in $S^{3}$ and $P$ is a bridge sphere for $L$ with $|P \cap L| \geq 6$. If $Q$ is another bridge sphere for $L$ such that $Q$ is not equivalent to $P$, then $d(P, L) \leq|Q \cap L|-2$, where $d(P, L)$ denotes the distance of the bridge sphere $P$.

By the above theorem, we can improve a result of Tomova [8, Corollary 10.7] in the case of bridge sphere.

Corollary 1.2. If $P$ is a bridge sphere for a link $L$ such that

$$
d(P, L)>|P \cap L|-2,
$$

then the minimal bridge sphere for $L$ is unique up to isotopy transverse to $P \cap L$.

\section{Definitions and notations}

Let $M$ be a closed orientable 3-manifold, $\gamma$ a union of mutually disjoint arcs or simple closed curves properly embedded in $M, F$ a surface embedded in $M$, which is in general position with respect to $\gamma$. A surface $D$ in $M$ is a $\gamma$-disk, if $D$ is a disk intersecting $\gamma$ in at most one transverse point. Let $\ell(\subset F)$ be a simple closed curve with $\ell \cap \gamma=\emptyset$. We say that $\ell$ is $\gamma$-inessential if $\ell$ bounds a $\gamma$-disk in $F$, and $\ell$ is $\gamma$-essential if it is not $\gamma$-inessential. We say that a surface $D$ is a $\gamma$-compressing disk for $F$ if; $D$ is a $\gamma$-disk, and $D \cap F=\partial D$, and $\partial D$ is a $\gamma$-essential simple closed curve in $F$. Let $F_{1}, F_{2}$ be surfaces in $M$ which are in general position with respect to $\gamma$. We say that $F_{1}$ and $F_{2}$ are $\gamma$-parallel if they co-bound a 3-manifold homeomorphic to $F_{1} \times[0,1]$ intersecting $\gamma$ in vertical arcs, where $F_{1}=F_{1} \times\{0\}$ and $F_{2}=F_{1} \times\{1\}$. We say that $F_{1}$ and $F_{2}$ are $\gamma$-isotopic if there exists an isotopy from $F_{1}$ to $F_{2}$ so that $F_{1}$ remains transverse to $\gamma$ throughout the isotopy.

\subsection{Handlebodies containing trivial arcs}

Let $H$ be a genus- $g(\geq 0)$ handlebody. If $g>1$, spine $\Sigma_{H}$ of $H$ is a 1-complex contained in the interior of $H$, which is a strong deformation retract of $H$, where each vertex of $\Sigma_{H}$ has valence three. Note that for a genus-0 handlebody (the 3 -ball), we let the spine be a point in the interior of the 3 -ball, and for a genus- 1 handlebody (solid torus), we let the spine be a core circle of the solid torus. We say that a set of $n$ arcs $\left\{t_{1}, \ldots, t_{n}\right\}$ properly in embedded in $H$ is a set of trivial $n$ arcs if $t_{1} \cup \cdots \cup t_{n}$ is parallel to $\partial H$. Let $H$ be a handlebody and $\tau=\left\{t_{1}, \ldots, t_{n}\right\}$ a set of trivial $n$ arcs in $H$. Then $\tau$ can be isotoped in $H$ so that the projection from $\partial H \times[0,1)$ to $[0,1)$ has exactly one critical point in each $t_{i}$. For the pair $(H, \tau)$, we let the spine $\Sigma_{(H, \tau)}$ be the union of $\Sigma_{H}$ together with a collection of vertical arcs $\alpha_{1}, \ldots, \alpha_{n}$, where one endpoint of each $\alpha_{i}$ lies on the critical point of $t_{i}$, and the other endpoint lies on $\Sigma_{H}$. 


\subsection{Bridge decompositions}

It is well known that every closed orientable 3 -manifold $M$ has a genus- $g$ Heegaard splitting for some $g(\geq 0)$, i.e., $M=A \cup_{P} B$, where $A$ and $B$ are genus$g$ handlebodies in $M$ such that $M=A \cup B$ and $A \cap B=\partial A=\partial B=P$. Let $L$ be a link in $M$. We say that $\left(A, \tau_{A}\right) \cup_{P}\left(B, \tau_{B}\right)$ is a $(g, n)$-bridge decomposition (or bridge decomposition for short) for the pair $(M, L)$ if $P$ separates $(M, L)$ into two components $\left(A, \tau_{A}\right)$ and $\left(B, \tau_{B}\right)$ where $\tau_{A}=L \cap A\left(\operatorname{resp} . \tau_{B}=L \cap B\right)$ is a set of trivial $n \operatorname{arcs}$ in $A$ (resp. $B)$. Then we say that $P$ is a $(g, n)$-bridge surface (or a bridge surface for short). It is known that each $(M, L)$ has a $(g, n)$-bridge decomposition for some $g$ and $n$. (For a detailed discussion, see [3, Lemma 2.1].)

Given a $(g, n)$-bridge decomposition $\left(A, \tau_{A}\right) \cup_{P}\left(B, \tau_{B}\right)$ for $(M, L)$, there are three ways to create new bridge surfaces for $(M, L)$ : (1) adding dual onehandles disjoint from $L$ (stabilizing), (2) adding dual one-handles where one of them has an arc of $L$ as its core (meridionally stabilizing), and (3) introducing a pair of a canceling minimum and maximum for $L$ (perturbing) (for details, see [8, Figure 15]). We say that another bridge surface $Q$ is equivalent to $P$ if $Q$ is $L$-isotopic to a copy of $P$ which may have been stabilized, meridionally stabilized and perturbed.

\subsection{Sweep-outs}

Let $L$ be a link in a closed orientable 3 -manifold $M$. Suppose $\left(A, \tau_{A}\right) \cup_{P}$ $\left(B, \tau_{B}\right)$ is a bridge decomposition for $(M, L)$. From the definition of a spine, one can construct a map $f: M \rightarrow[-1,1]$ such that $f^{-1}(-1)$ is a spine of $\left(A, \tau_{A}\right), f^{-1}(1)$ is a spine of $\left(B, \tau_{B}\right)$ and $f^{-1}(s)$ is a surface which is $L$-parallel to the bridge surface $P$ for each $s \in(-1,1)$. This map is called a sweep-out induced from $\left(A, \tau_{A}\right) \cup_{P}\left(B, \tau_{B}\right)$. For each $s \in(-1,1)$, we put $P_{s}=f^{-1}(s)$, $A_{s}=f^{-1}([-1, s])$ and $B_{s}=f^{-1}([s, 1])$.

\subsection{Curve complexes}

Let $S$ be a compact orientable surface with genus $g$ and $p$ punctures. The curve complex $\mathcal{C}(S)$ is defined as follows: the vertices of $\mathcal{C}(S)$ are the isotopy classes of essential simple closed curves on $S$, and a collections of $k+1$ vertices form a $k$-simplex of $\mathcal{C}(S)$ if they can be realized by disjoint curves in $S$. For two vertices $x, y$ of $\mathcal{C}(S)$, we define the distance $d(x, y)$ between $x$ and $y$ as the minimal number of 1-simplexes of a simplicial path in $\mathcal{C}(S)$ joining $x$ and $y$. Let $X, Y$ be subsets of the vertices of $\mathcal{C}(S)$. Then we define $d(X, Y)=$ $\min \{d(x, y) \mid x \in X, y \in Y\}$.

Let $H$ be a handlebody and $\tau$ a set of trivial $n$ arcs in $H$. Then $\mathcal{D}(H \backslash \tau)$ denotes the subset of $\mathcal{C}(\partial H \backslash \partial \tau)$ consisting of the vertices with representatives bounding disks in $H \backslash \tau$. Suppose that $M$ is a closed orientable 3-manifold containing a link $L$, and $\left(A, \tau_{A}\right) \cup_{P}\left(B, \tau_{B}\right)$ is a bridge decomposition for $(M, L)$. 
Then the distance $d(P, L)$ of $\left(A, \tau_{A}\right) \cup_{P}\left(B, \tau_{B}\right)$ is defined by $d\left(\mathcal{D}\left(A \backslash \tau_{A}\right)\right.$, $\left.\mathcal{D}\left(B \backslash \tau_{B}\right)\right)$.

Let $B^{3}$ be a 3 -ball, and $\tau$ a set of trivial $n$ arcs in $B^{3}$ with $n \geq 3$.

Lemma 2.1. Let $D$ be a $\gamma$-compressing disk in $B^{3}$. Then for a $\tau$-essential simple closed curve $\ell \subset \partial\left(B^{3} \backslash \partial \tau\right)$ which is disjoint from $\partial D, d\left(\mathcal{D}\left(B^{3} \backslash \tau\right), \ell\right) \leq$ 1 .

Proof. If $D \cap \tau=\emptyset$, then clearly Lemma 2.1 holds. Suppose $D \cap \tau$ consists of one point. Then $D$ separates $\left(B^{3}, \tau\right)$ into two components $\left(B_{1}^{3}, \tau_{1}\right)$ and $\left(B_{2}^{3}, \tau_{2}\right)$ where $\ell \subset \partial B_{1}^{3} \backslash \partial \tau_{1}$. Since $D$ is a $\tau$-compressing disk, $\tau_{2}$ consists of at least two components. Hence there is a compressing disk $D^{\prime}\left(\subset B_{2}^{3}\right)$ for $\partial B_{2}^{3} \backslash \partial \tau_{2}$. Further, by $\tau_{2}$-isotopy of $B_{2}^{3}$, we may suppose that $D^{\prime}$ is disjoint from the image of $D$. Hence we may regard $D^{\prime}$ a compressing disk in $B^{3} \backslash \tau$. Since $\ell$ is disjoint from $D^{\prime}$, we have $d\left(\partial D^{\prime}, \ell\right) \leq 1$, which implies $d\left(\mathcal{D}\left(B^{3} \backslash \tau\right), \ell\right) \leq 1$

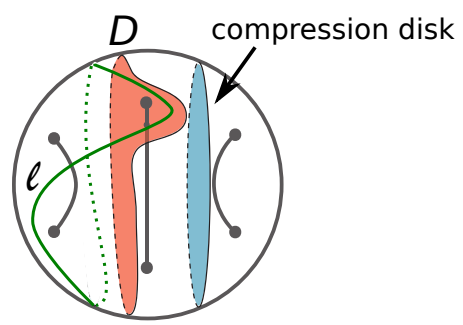

FIGURE 1

\section{Proof of main results}

Let $L$ be a link in $S^{3}$. Let $\left(A, \tau_{A}\right) \cup_{P}\left(B, \tau_{B}\right)$ be a $\left(0, n_{1}\right)$-bridge decomposition $\left(n_{1} \geq 3\right)$ for $\left(S^{3}, L\right)$, and $f$ a sweep-out induced from $\left(A, \tau_{A}\right) \cup_{P}\left(B, \tau_{B}\right)$. Let $\pi_{P}$ be the projection map from $P \times(-1,1)$ to $P$.

Suppose that $Q$ is a $\left(0, n_{2}\right)$-bridge surface $\left(n_{2} \geq 3\right)$ for $\left(S^{3}, L\right)$ which is not equivalent to $P$. Then, by [6, Theorem 3.1 and Lemma 4.3], $Q$ can be $L$-isotoped so that $\left.f\right|_{Q}$ is Morse, and for every $s \in(-1,1), P_{s} \cap Q$ contains a curve that is $L$-essential in $P_{s}$. Moreover, as in the proof of $[6$, Theorem $4.2]$, either $d(P, L) \leq 1$ or there exists an interval $\left[s_{-}, s_{+}\right]$, where $s_{-}<s_{+}$are critical values for $\left.f\right|_{Q}$ such that

(1) for every $s \in\left(s_{-}, s_{+}\right)$, each component of $P_{s} \cap Q$ which is $L$-essential in $P_{s}$ does not bound a disk in $Q$, and

(2) for a small $\epsilon, P_{s_{-}-\epsilon} \cap Q$ contains a curve that is $L$-essential in $P_{s_{-}-\epsilon}$ and bounds a disk in $A_{s_{-}-\epsilon}$, and $P_{s_{+}+\epsilon} \cap Q$ contains a curve that is $L$-essential in $P_{s_{+}+\epsilon}$ and bounds a disk in $B_{s_{+}+\epsilon}$. 
Hence we have:

$$
\begin{gathered}
\text { if } d(P, L) \geq 2, \text { there exists an interval }\left[s_{-}, s_{+}\right] \text {satisfying } \\
\text { the conditions (1) and }(2) .
\end{gathered}
$$

We show that in $(*)$, the conclusion can be improved if $d(P, L)>2$. Namely:

Lemma 3.1. Let $Q$ be as above. If $d(P, L)>2$, there exists a subinterval $\left[s_{-}^{\prime}, s_{+}^{\prime}\right] \subset\left[s_{-}, s_{+}\right]$, where $s_{-}^{\prime}<s_{+}^{\prime}$ are critical values for $\left.f\right|_{Q}$ such that

(i) for every $s^{\prime} \in\left(s_{-}^{\prime}, s_{+}^{\prime}\right)$, each component of $P_{s^{\prime}} \cap Q$ which is L-essential in $P_{s^{\prime}}$ does not bound an $L$-disk in $Q$, and

(ii) for a small $\epsilon, P_{s^{\prime}-\epsilon} \cap Q$ contains a curve that is L-essential in $P_{s^{\prime}-\epsilon}$ and bounds an L-disk in $A_{s_{-}^{\prime}-\epsilon}$, and $P_{s_{+}^{\prime}+\epsilon} \cap Q$ contains a curve that is L-essential in $P_{s_{+}^{\prime}+\epsilon}$ and bounds an $L$-disk in $B_{s_{+}^{\prime}+\epsilon}$.

Proof. Suppose, for a contradiction, that for every $s \in\left(s_{-}, s_{+}\right)$, there exists a component of $P_{s} \cap Q$ which is $L$-essential in $P_{s}$ and bounds a $L$-disk in $Q$. Note that a disk in $Q$ is an $L$-disk. Hence by the above condition (1), we obtain that there exists a critical value $s^{*}$ (possibly $s^{*}=s_{-}$or $s_{+}$) such that for a small $\epsilon, P_{s^{*}-\epsilon} \cap Q$ contains a curve bounding a $L$-disk $D_{A}$ in $A_{s^{*}-\epsilon}$, and $P_{s^{*}+\epsilon} \cap Q$ contains a curve bounding a $L$-disk $D_{B}$ in $B_{s^{*}+\epsilon}$. Note that $d\left(\pi_{P}\left(\partial D_{A}\right), \pi_{P}\left(\partial D_{B}\right)\right) \leq 1$. (Recall that $\pi_{P}$ be the projection map from $P \times(-1,1)$ to $P$.) Hence by regarding $D_{A}$ as $D$ and $\pi_{P}\left(\partial D_{B}\right)$ as the $\ell$ in Lemma 2.1, we have $d\left(\mathcal{D}\left(A \backslash \tau_{A}\right), \pi_{P}\left(\partial D_{B}\right)\right) \leq 1$. Analogously we have $d\left(\mathcal{D}\left(B \backslash \tau_{B}\right), \pi\left(\partial D_{B}\right)\right) \leq 1$. These together with a triangle inequality $d_{P}\left(\mathcal{D}\left(A \backslash \tau_{A}\right), \mathcal{D}\left(B \backslash \tau_{B}\right)\right) \leq d\left(\mathcal{D}\left(A \backslash \tau_{A}\right), \pi_{P}\left(\partial D_{B}\right)\right)+d\left(\pi_{P}\left(\partial D_{B}\right), \mathcal{D}\left(B \backslash \tau_{B}\right)\right)$ imply that $d(P, L) \leq 2$, a contradiction. Hence, there exists a component of $P_{s^{\prime}} \cap Q$ which is $L$-essential in $P_{s^{\prime}}$ and does not bound a $L$-disk in $Q$. It is easy to see that this implies Lemma 3.1(i) holds. The conclusion (ii) follows from the conclusion (i) and the above condition (1).

Proof of Theorem 1.1. By Lemma 3.1, either (I)d(P,L) $\leq 2$ or (II)there exists an interval $\left[s_{-}^{\prime}, s_{+}^{\prime}\right]$, where $s_{-}^{\prime}<s_{+}^{\prime}$ are critical values for $\left.f\right|_{Q}$ satisfying (i) and (ii) of Lemma 3.1. If $d(P, L) \leq 2$, then since $n_{2} \leq 3$, the conclusion of Theorem 1.1 holds. Hence we consider the case (II). Let $C$ be the union of the components of $P_{s_{-}^{\prime}+\epsilon} \cap Q$ and $P_{s_{+}^{\prime}-\epsilon} \cap Q$ which are $L$-essential on $Q$. Since $Q$ is connected, there is a component, say $Q^{\prime}$, of $Q \backslash C$ such that $\partial Q^{\prime} \cap P_{s_{-}^{\prime}+\epsilon} \neq \emptyset$ and $\partial Q^{\prime} \cap P_{s_{+}^{\prime}-\epsilon} \neq \emptyset$. Note that each component of $\partial Q^{\prime} \cap P_{s_{-}^{\prime}+\epsilon}$ and $\bar{\partial} Q^{\prime} \cap P_{s_{+}^{\prime}-\epsilon}$ bounds an at least twice punctured disk in $\left(Q \backslash Q^{\prime}\right) \backslash L$ because each component of $\partial Q^{\prime} \cap P_{s_{-}^{\prime}+\epsilon}$ and $\partial Q^{\prime} \cap P_{s_{+}^{\prime}-\epsilon}$ is $L$-essential on $Q$. Hence $\chi\left(Q^{\prime} \backslash L\right) \geq$ $\chi(Q \backslash L)+2$. Let $c_{-}$(resp. $\left.c_{+}\right)$be a component of $c l\left(Q^{\prime}\right) \cap P_{s_{-}^{\prime}+\epsilon}$ (resp. $\left.c l\left(Q^{\prime}\right) \cap P_{s_{+}^{\prime}-\epsilon}\right)$. Hence by using arguments as in the proof of $[6$, Theorem $4.2], d\left(\pi_{P}\left(c_{-}\right), \pi_{P}\left(c_{+}\right)\right) \leq-\chi\left(Q^{\prime} \backslash L\right)$. By (ii) of Lemma 3.1, $\pi_{P}\left(c_{-}\right)$(resp. $\left.\pi_{P}\left(c_{+}\right)\right)$is disjoint from an $L$-compressing disk in $A$ (resp. $B$ ). By Lemma 2.1, 


$$
\begin{aligned}
& d\left(\mathcal{D}\left(A \backslash \tau_{A}\right), \pi_{P}\left(c_{-}\right)\right) \leq 1 \text { and } d\left(\pi_{P}\left(c_{+}\right), \mathcal{D}\left(B \backslash \tau_{B}\right)\right) \leq 1 . \text { Hence, we have } \\
& d(P, L) \leq d\left(\mathcal{D}\left(A \backslash \tau_{A}\right), \pi_{P}\left(c_{-}\right)\right)+d\left(\pi_{P}\left(c_{-}\right), \pi_{P}\left(c_{+}\right)\right)+d\left(\pi\left(c_{+}\right), \mathcal{D}\left(B \backslash \tau_{B}\right)\right) \\
& \leq 1-\chi\left(Q^{\prime} \backslash L\right)+1 \\
&=-\chi(Q \backslash L) .
\end{aligned}
$$

This completes the proof of Theorem 1.1.

Proof of Corollary 1.2. Let $Q$ be a minimal bridge sphere for a link $L$. Suppose that $Q$ not equivalent to $P$. Then, by Theorem 1.1, $d(P, L) \leq|Q \cap L|-2=$ $|P \cap L|-2$, a contradiction.

Acknowledgments. I would like to thank Professor Tsuyoshi Kobayashi for many helpful advices and comments. I would also like to thank Yeonhee Jang for her valuable advices and informations. I gratefully appreciate the financial support from Japan Society for the Promotion of Science (JSPS). Finally I would like to thank the referee for careful reading of the first version of the paper.

\section{References}

[1] D. Bachman and S. Schleimer, Distance and bridge position, Pacific J. Math. 219 (2005), no. 2, 221-235.

[2] K. Hartshorn, Heegaard splittings of Haken manifolds have bounded distance, Pacific J. Math. 204 (2002), no. 1, 61-75.

[3] C. Hayashi and K. Shimokawa, Thin position of a pair (3-manifold, 1-submanifold), Pacific J. Math. 197 (2001), no. 2, 301-324.

[4] J. Hempel, 3-manifolds as viewed from the curve complex, Topology 40 (2001), no. 3, 631-657.

[5] Y. Jang, Distance of bridge surfaces for links with essential meridional spheres, Pacific J. Math. 267 (2014), no. 1, 121-130.

[6] J. Johnson and M. Tomova, Flipping bridge surfaces and bounds on the stable bridge number, Algebr. Geom. Topol. 11 (2011), no. 4, 1987-2005.

[7] M. Scharlemann andn M. Tomova, Alternate Heegaard genus bounds distance, Geom. Topol. 10 (2006), 593-617.

[8] M. Tomova, Multiple bridge surfaces restrict knot distance, Algebr. Geom. Topol. 7 (2007), 957-1006.

Department of Mathematics Education

Aichi University of EDUCATion

1 Hirosawa, Igaya-Cho, Kariya, Aichi 448-8542, Japan

E-mail address: ayakoido@auecc.aichi-edu.ac.jp 